\title{
Efficacy of Dual Parenteral Artemisinin-Based Combination Therapy for Cerebral Malaria in a Tripple Blinded Randomized Control Trial in Nigerian Children: DUAL PACT TRIAL
}

\section{Michael Abel Alao ( $\sim$ mikevikefountains@gmail.com )}

UCH: University College Hospital Ibadan https://orcid.org/0000-0003-0109-4435

Adebola Emmanuel Orimadegun

University of Ibadan College of Medicine

Olayinka Rasheed Ibrahim

Federal Medical Centre Katsina

Abayomi 0 Oyenuga

University of Minnesota Medical Center Fairview: University of Minnesota Health

Adanze Onyenonachi Asinobi

UCH: University College Hospital Ibadan

\section{Daniel Adedosu Gbadero}

Bowen University College of Health Sciences

Ifeoma Joy Okoye

UNTH: University of Nigeria Teaching Hospital

Emmanuel Okechukwu Nna ( $\nabla$ e.nna@themping.org )

Molecular Pathology Institute https://orcid.org/0000-0001-6791-2336

\section{Research Article}

Keywords: Cerebral malaria, artesunate, quinine, randomized control trial, blinding

Posted Date: March 15th, 2021

DOl: https://doi.org/10.21203/rs.3.rs-325850/v1

License: (c) (i) This work is licensed under a Creative Commons Attribution 4.0 International License. Read Full License 


\section{Abstract}

\section{Background:}

Evidence exists as to the criticality of the first 24 hours in the management of cerebral malaria. This could serve as a potential safety net for improved outcomes with prompt treatment and utilization of effective therapy. The morbidity and the mortality rate (35\%) with the current parenteral monotherapy for the initial treatment of cerebral malaria is unacceptably high. Combination therapy and a shorter course of effective medication have been shown to improve outcomes in human subjects in treatment of other diseases. This study outlines a protocol to conduct a triple blinded randomized control trial on cerebral malaria using dual parenteral medications compared to the current standard of monotherapy.

Methods: This is a randomized control triple blinded trial consisting of an intervention and 2 control arms. Eligible and assenting children aged 6 months to 17 years will be recruited from 4 tertiary hospitals by random selection from the list of tertiary hospitals in Nigeria. Subjects will be concealed and allocated in parallel into three arms using random numbers generated from GraphPad Prism (version 9) by a Clinical Pharmacologist who has no link with the investigators, the patients, or the statistician. The parasite load and clearance, fever defervesence, C reactive protein, procalcitonin, blood glucose, electrocardiograms and time to regain consciousness will be monitored. The socio-demographic and clinical details of all patients will be documented. The study will be conducted in line with Declaration of Hesinki 2013. Appropriate statistical tools will be used for data collection and analysis.

\section{Discussion:}

The outcome of this analysis will give insight into the efficacy of dual parenteral antimalaria in the treatment of cerebral malaria among Nigerian children compared with standard of care. The safety profile of this intervention would also be highlighted. This may help inform Physicians on the optimal treatment for cerebral malaria to improve outcomes, prevent development of resistance, recrudescence, treatment failure and stimulate interest of pharmaceutical companies in the development of newer formulations of dual parenteral antimalarial combination therapies.

Trial registration: This study was registered in the Pan Africa Clinical Trial Registry (PACTR202102893629864).

\section{Background And Rationale \{6a\}}

Malaria remains a global health problem and a threat to human survival in over one hundred countries of the world.[1-6] It is one of the leading causes of morbidity and mortality among the under-five in the subSaharan Africa.[1] In spite of the concerted efforts and huge resources dedicated to malaria eradication, its tales of woes remains strong and visible. $[1,3,7]$ The socio-economic burden of malaria in endemic 
communities, especially in the sub-Saharan Africa is still colossal. It is a major source of drain to the scarce resources in low and middle-income countries of the world (LMIC).[7]

Reports from previous studies $[1,2,7]$ have shown that severe/complicated malaria carries $100 \%$ mortality if untreated. The most critical moment determining outcomes of severe malaria is the first twenty-four hours from disease onset; often characterised by multiple organ dysfunction syndrome.[1, 2] However, current treatment regimens use monotherapy that provides opportunity for the development of drug resistance and suboptimal outcome.[7] Current mortality from severe malaria ranges between 22.5 to $34.7 \%$ in Africa and Asia, respectively.[1, 8-10] Although there has been some improvement in outcomes $[1,8,11]$, the high morbidity and mortality in the endemic countries are still concerning.

The current treatment for uncomplicated malaria advocates combination therapy of artemisinin-based regimens, the question is whether the use of dual artemisinin-based parenteral antimalarial compared to the current recommendation of monotherapy in terms of parasite clearance, fever resolution, and recovery of consciousness in cerebral malaria would improve outcome. We hypothesized that initiation of appropriate, optimal, and adequate dual parenteral anti-malaria therapy within the first 24 hours may positively impact outcomes. $[9,10]$ This protocol, therefore, provides a workflow to compare the efficacy of dual parenteral ACT-based regimen with the current gold standard of artesunate or quinine regimen alone in a randomized control trial.

\section{Hypothesis}

Initiation of appropriate, optimal, and adequate dual parenteral anti-malaria (Artesunate \& Quinine) within the first 24 hours of diagnosis of cerebral malaria will result in improved clinical outcomes compared to monotherapy (artesunate only, or quinine only).

\section{Research Problem}

Severe malaria with multiple organ dysfunction stands at the top of the list of causes of under-five mortality in sub-Saharan Africa.[1] The morbidity and mortality rate of $35 \%$ with the current monotherapy is unacceptably high.[8] There is a need for modification of current treatment regimen to improve outcomes, prevent development of resistance, recrudescence, and treatment failure.

\section{Rationale for the study}

Combination therapy and a shorter course of effective medication in treatment of diseases have been shown to improve outcomes in Human Immunodeficiency virus AID (HIV-AID) infection, tuberculosis, acute pyogenic infections and epilepsy.[12-15] If the critical time for severe malaria treatment is the first 24 hour of illness, aggressive therapy targeted during this time using a synergistic combination of parenteral artesunate-quinine has a potential for rapid parasite clearance and reduce morbidity and mortality. This trial is feasible because of the long-standing biosafety profiles of artesunate and quinine. We are optimistic that the results of this trial may stimulate interest of pharmaceutical companies in the development of newer formulations of parenteral antimalarial combination therapies, this could improve 
the availability and administration of treatment within the first 24 hours and consequently improve the health outcomes in patients diagnosed with cerebral malaria.

\section{Objectives $\{7\}$}

\section{General objective:}

The general objective is to determine the efficacy of dual parenteral artesunate - quinine combination regimen against the monotherapy of artesunate or quinine in the treatment of cerebral malaria.

\section{Specific objectives:}

To determine:
a. Survival rate from cerebral malaria on dual parenteral antimalarial compared to monotherapies,
b. The parasite clearance rate,
c. Fever defervescence,
d. Time to recovery of consciousness,
e. Time to conversion of dual parenteral treatment to full dose of enteral artemisinin-based combination therapy,
f. Side effect profile of interventions (treatment and control arms), and
g. Neurologic status at the fourth week.

\section{Trial design $\{8\}$}

This will be triple blinded, parallel randomized control trial consisting of one treatment/experimental arm and two control arms. Patient allocation will be in the ratio 1:1:1 of $A, B, C$ arms. The allocation of patients into the study arms will be concealed.

\section{Methods: Participants, Interventions And Outcomes}

The participants' flow chart from invitation through enrolment, allocation to trial is shown in Figure 1

\section{Study setting $\{9\}$}

This study is a multicenter study involving 4 tertiary hospitals (one private and three public) located in three of the six geopolitical regions in Nigeria.[16, 17] The selected tertiary hospital includes University College Hospital Ibadan. Bowen University Teaching Hospital Ogbomoso, The Federal Medical Centre Kastina, Kastina state and The University of Maiduguri Teaching Hospital, Maiduguri.

All the facilities have the human capacity to manage critically ill children. Other details are given: Administrative details (Figure 2) and Trial Site locations (Figure 3). 


\section{Eligibility criteria $\{10\}$}

\section{Inclusion Criteria}

1. Children between the ages of 6 months to 17 years as at the last birthday.

2. Children with unarousable coma lasting more than 30 minutes in the presence of demonstrable peripheral asexual $P$. falciparum parasitaemia.

3. Children who develop deterioration in their level of consciousness after admission and demonstrate asexual Plasmodium falciparum in the peripheral blood.

\section{Exclusion Criteria}

1. Children with positive biochemical and/or microbiological CSF result consistent with a diagnosis of meningitis.

2. Children who have intracranial infections or intracranial space occupying lesion.

3. Children with previous neurological deficitt either focal or global.

4. Children whose caregiver declined granting consent for their participation in the study.

\section{Who will take informed consent? \{26a\}}

Prior to recruitment, the research would be explained to parents/caregivers by the investigator who will obtain written informed consent. Parents/caregivers will be informed of their freedom to refuse to take part in the study without any negative consequences to them or their wards in the course of treatment.

\section{Additional consent provisions for collection and use of participant data and biological specimens \{26b\}}

Additional consent would be obtained for data availability for secondary analysis and for ancillary studies on biological specimen collected for the research.

\section{Interventions}

\section{Explanation for the choice of comparators $\{6 b\}$}

Intravenous artesunate alone and intravenous quinine alone would be used as comparator in this study. These are the current standard treatments for cerebral malaria by the World Health Organization. In order to facilitate blinding, the monotherapy groups (controls) will also receive intravenous dextrose water or water for injection to maintain similarity of treatment administration with the dual-therapy group (the treatment arm). 
This arm will receive intravenous artesunate at $3 \mathrm{mg} / \mathrm{kg}$ if weight is less than $20 \mathrm{~kg}$ or $2.4 \mathrm{mg} / \mathrm{kg}$ if weight is greater than $20 \mathrm{~kg}$ at 0,12 , and 24 hours and subsequently a daily dose of $1.2 \mathrm{mg} / \mathrm{kg} /$ day until conscious. Additionally, intravenous $10 \%$ dextrose water will be given in lieu of intravenous quinine.

\section{Arm C}

This group will receive intravenous quinine at a dose of $20 \mathrm{mg} / \mathrm{kg}$ loading dose and subsequently $10 \mathrm{mg}$ $/ \mathrm{kg} 8$ hourly for $72 \mathrm{hr}$. However, if the patient remains unconscious the dose would be reduced to 7.5 $\mathrm{mg} / \mathrm{kg}$ 8hrly until he/she regains consciousness. Additionally, water for injection will be given in lieu of intravenous artesunate.

\section{Intervention description $\{11 \mathrm{a}\}$}

The treatment arm (Arm A) will receive a combination of parenteral quinine and artesunate. A clinical pharmacologist will deliver the allocated treatment to the clinician for administration.

This group will receive a combination of intravenous quinine and artesunate. The quinine will be given at a loading dose of $20 \mathrm{mg} / \mathrm{kg}$ and subsequently $10 \mathrm{mg} / \mathrm{kg} 8$ hourly for $72 \mathrm{hr}$. However, if the patient remains unconscious the dose would be reduced to $7.5 \mathrm{mg} / \mathrm{kg} 8 \mathrm{hrly}$ until he/she regain consciousness. In parallel, intravenous artesunate at $3 \mathrm{mg} / \mathrm{kg}$ if weight is less than $20 \mathrm{~kg}$ or $2.4 \mathrm{mg} / \mathrm{kg}$ if weight is greater than $20 \mathrm{~kg}$ at 0,12 , and 24 hours and subsequently a daily dose of $1.2 \mathrm{mg} / \mathrm{kg} /$ day until conscious.

\section{All the groups}

All the three groups would receive enteral artemisinin-based combination therapy (ACT) as soon as they regain consciousness and can tolerate the full course of oral ACT in line with WHO recommendation.

\section{Criteria for discontinuing or modifying allocated interventions $\{11 \mathrm{~b}\}$}

1. Participants who regained full recovery after initial treatment.

2. Participants who also wish to exit after due counselling.

3. Participants with intractable hypoglycaemia despite maintenance at $12.5 \%$ glucose.

4. Participants with dysrhythmias would be unblinded.

5. Participants who develop commodities of severe malaria that could confound the measurable outcomes such as uraemic encephalopathy.

6. Participants with new disease beside cerebral malaria or updated clinical history related to exclusion criteria 


\section{Strategies to improve adherence to interventions $\{11 \mathrm{c}\}$}

a. Prior to recruitment, parents/caregivers of study participants will be educated on the complication of severe malaria to gain their support in the research.

b. All study participants will enjoy social support throughout the hospital stay.

c. The possible side effect profile of artesunate and quinine would be discussed with the parents/caregivers at the enrolments for the study.

d. The cost of treatment in relation to cerebral malaria and some miscellaneous costs will also be borne by the sponsor(s) as a responsibility toward the patient.

\section{Relevant concomitant care permitted or prohibited during the trial $\{11 \mathrm{~d}\}$}

1. Concomitant treatment for septicaemia, severe anaemia, AKI stages 1 and 2 , hypoglycaemia will be permitted.

2. Treatments for other comorbidities of severe malaria which do not confound the protocol and measured outcomes will be permissible.

\section{Provisions for post-trial care $\{30\}$}

The participants with neurologic deficit as a result of the disease will be followed up by the Paediatric Neurologist for continuum of care.

\section{Outcomes $\{12\}$}

\section{The primary outcome}

1. The primary endpoint will be a $10 \%$ reduction in mortality between the experimental arm and the controls.

\section{Secondary outcomes}

i. The proportion of survival in the experimental and the control arms.

ii. The proportion of clinical success treatment outcome as defined by a composite end point of regaining full consciousness/GCS/MGCS of $>11$ or death at 24 hour of intervention and the status of asexual form of malaria parasite (indicated by either present or absent) at 48hours of intervention.

iii. The proportion of participants who regained full consciousness or the proportion with Glasgow/modified Glasglow coma score (GCS/MGCS) of more than 11 at 12 hours from the first dose of intervention.

iv. The proportion of participants with fever defervescence defined as time from the first dose of intervention to the attainment and sustenance of axillary temperature of $<37.5^{\circ} \mathrm{C}$ or less than 38.0 ${ }^{\circ} \mathrm{C}$ for rectal temperature. 
v. The proportion of participants with $90 \%$ or more reduction in Plasmodium falciparum parasite density at 12 hours of intervention.

vi. Time to parasite clearance in each of the treatment arms defined as time from initiation of therapy to total and sustained disappearance of asexual form of falciparum for at least 24hours

vii. Presence of neurologic deficits at the fourth week post intervention in each of the arms. Neurology deficit is defined as the presence of impaired cognitive, motor or cranial nerve palsy and convulsions.

viii. Time to convert to full dose of enteral artemisinin-based combination therapy.

ix. Side effect profile of medication in each arm. These would include dysthymias, cinchonism and visual impairment.

x. Time to normalisation or at least $50 \%$ reduction in levels of inflammatory markers such as $C$ reactive protein, procalcitonin, serum lactate dehydrogenase (LDH)

\section{Participant timeline $\{13\}[18]$}




\begin{tabular}{|c|c|c|c|c|c|c|c|c|}
\hline & \multicolumn{8}{|c|}{ STUDY PERIOD } \\
\hline & \multirow{2}{*}{$\begin{array}{c}\text { Enrolment } \\
t_{1}\end{array}$} & \multirow{2}{*}{$\begin{array}{c}\text { Allocation } \\
0\end{array}$} & \multicolumn{5}{|c|}{ Post-allocation } & \multirow{2}{*}{$\begin{array}{c}\text { Close } \\
\text { out }\end{array}$} \\
\hline TIMEPOINT ${ }^{\star \star}$ & & & $12 \mathrm{hs}$ & 24hrs & $48 \mathrm{hrs}$ & $72 \mathrm{hrs}$ & 28d & \\
\hline \multicolumn{9}{|l|}{ ENROLMENT: } \\
\hline Eligibility screen & $\mathrm{x}$ & & & & & & & \\
\hline Informed consent & $\mathrm{X}$ & & & & & & & \\
\hline $\begin{array}{c}\text { Amthropometry } \\
\text { measurement }\end{array}$ & $\mathrm{X}$ & & & & & & & \\
\hline $\begin{array}{l}\text { Clinical History } \\
\text { and detailed CNS }\end{array}$ & $\mathrm{x}$ & & & & & $\mathrm{X}$ & & \\
\hline Allocation & & $\mathrm{X}$ & & & & & & \\
\hline \multicolumn{9}{|l|}{ INTERVENTIONS: } \\
\hline \multicolumn{9}{|l|}{ [Intervention A] } \\
\hline \multicolumn{9}{|l|}{ [Intervention B] } \\
\hline \multicolumn{9}{|l|}{ [Intervention C] } \\
\hline \multicolumn{9}{|l|}{ ASSESSMENTS: } \\
\hline $\begin{array}{c}\text { [List baseline } \\
\text { variables] }\end{array}$ & $x$ & $x$ & & & & & & \\
\hline $\begin{array}{r}\text { [List outcome } \\
\text { variables] }\end{array}$ & & & & $\mathrm{X}$ & & $\mathrm{X}$ & etc. & $X$ \\
\hline $\begin{array}{r}\text { [List other data } \\
\text { variables] }\end{array}$ & & & $\mathrm{X}$ & $X$ & $\mathrm{X}$ & $\mathrm{X}$ & etc. & $\mathrm{X}$ \\
\hline
\end{tabular}

\section{Sample size $\{14\}$}

The sample size for this study was calculated using G*Power calculator. Based on an effect size of $10 \%$ reduction in proportion of mortality due to cerebral malaria (at a prevalence of 35\%), a sample size of 716 has $90 \%$ power at the alpha level of significance 0.05 . Based on $1: 1$ allocation, the treatment arm will have 358 subjects while the control arms, each will have 179 subjects.

\section{Recruitment $\{15\}$}

Invitation: The caregivers/ parents of the eligible patients will be verbally invited during their hospital admission. The participant information sheet (PIS) will be explained to them in the language they understand.

Eligibility: Subjects will be assessed based on the eligibility criteria enumerated above. 
Enrolment: Eligible subjects will be enrolled into the study after giving written informed consent.

Informed Consent: Parents and caregivers will give a written informed consent during enrolment. This will be signed by Principal Investigator, the parent/caregiver, and a witness. A copy of informed consent will be retained by the parent, while a copy will be kept in the patient's file.

\section{Sampling technique}

In stage I: The list of the tertiary hospitals in Nigeria is the sample frame for the study. Four institutions have been selected from this list.

In Stage Il: an allocation sequence for the 3 arms will be generated using simple randomization from GraphPad Prism version 9. In the selected hospitals, patients with cerebral malaria, confirmed by a blood film malaria test but with a negative CSF result whose parents/caregivers give informed consented would be assigned to one of the 3 arms based on the assignment sequence.

\section{Allocation:}

Allocation concealment will be done by a Clinical pharmacologist who has no link with the investigators, the patients, or the statistician. Subjects will be allocated in parallel (concurrent) in to three arms using random numbers generated from GraphPad Prism (version 9) for the study.

1. Arm A: The intervention for parenteral artesunate and quinine.

2. Arm B: The parenteral quinine arm with intravenous water for injection.

3. Arm C: The standard of care of parenteral artesunate and intravenous 10\% Dextrose water.

\section{Assignment of interventions: Allocation}

\section{Sequence generation $\{16 \mathrm{a}\}$}

A random number will be generated using simple randomization from GraphPad Prism version 9 for patients being enrolled for the study.

\section{Concealment mechanism \{16b\}}

The random number generated by GraphPad Prism version 9 will be used to assign participants to either of the arms. This process will be done by the centrally located Clinical Pharmacologist who has no link with the other research team. Participants allocation to any of the treatment arms will be concealed from the investigators, the patients and the statistician (tripple blinding). The Clinical Pharmacologist will 
deliver allocated intervention (the treatment packaging is similar) to the clinician who will administer the intervention concurrently to eligible study participants.

\section{Implementation $\{16 \mathrm{c}\}$}

A Clinical Pharmacologist will be responsible for generating a random number and its allocation using GraphPad Prism version 9. He will equally be responsible for delivering randomized intervention to clinician for execution.

\section{Assignment of interventions: Blinding}

\section{Who will be blinded $\{17 \mathrm{a}\}$}

The treatment will be triple blinded. The investigator/clinician, parents/caregivers, and the statistician (outcome assessor) will not be aware (blinded) to the interventions.

\section{Procedure for unblinding if needed $\{17 b\}$}

When there is adverse event or features of toxicity of any of the drug of intervention, the clinical pharmacologist will be called to unblind the allocation since he is the only one who has access to the concealed allocation.

\section{Data collection and management}

\section{Plans for assessment and collection of outcomes $\{18 \mathrm{a}\}$}

Prior to commencement of the trial, a pilot study will be conducted at the University College Hospital Ibadan where the steering committee members are, alongside the focal leads from the other three collaborating centres in order to test the questionnaire and the protocol. Data from pilot study will be entered into Microsoft excel spread sheet uploaded into GraphPad Prism version 9 and check for appropriateness, completeness and adequacy. Observation from the dummy analysis will be used to improve the data entry and address other observed limitations.

A debriefing session would be held to review the research process and appropriate adjustment where needed to improve the quality of the instrument and enhance understanding of the protocol for better outcome. Subsequently the site Principal Investigators will step down such training to the collaborating centres.

Plans to promote participant retention and complete follow-up $\{18 \mathrm{~b}\}$ 
The study participants will be contacted via their phone to remind them of the next visit to the neurologist. The transportation fare will be borne by the research sponsor to facilitate retention on followup.

\section{Data management $\{19\}$}

Data obtained from the study will be entered into a password protected and encrypted institutional REDCap database. Only specific individuals from the collaborating centres will be given access to the database. All data from all the centres will be de-identified and managed through secure code. The data assessor (statistician) will be blinded to the data until the final stage of analysis.

\section{Confidentiality $\{27\}$}

All information collected in this study will be given code numbers, and no name will be recorded. This cannot be linked to the patients, parents or care providers in any way. Identifier will not be used in any publication or reports from the study.

\section{Plans for collection, laboratory evaluation and storage of biological specimens for genetic or molecular analysis in this trial/future use $\{33\}$}

This trial has no intention for genetic study on the respondent.

\section{Statistical methods for primary and secondary outcomes $\{20 \mathrm{a}\}$}

Data from this study will be analysed using the GraphPad Prism 9 (GraphPad Software 2365 Northside Dr. Suite 560 San Diego, CA 92108). The appropriate descriptive statistics will be used to present the socio-demographic characteristics of study participants. The comparison of categorical outcomes between the arms will be analysed using the Chi-squared or Fisher's exact tests, as appropriate, and presented as risk differences, risk ratios and $95 \%$ confidence intervals. Kaplan-Meier analysis and Cox regression models will be used to compare the recovery of consciousness, defervescence, and all-cause mortality. P-values $<0.05$ will be considered statistically significant for all analyses.

\section{Interim analyses $\{21 \mathrm{~b}\}$}

An interim analysis will be conducted by the outcome assesor (33\% into the sample collection) who will be blinded to the study. The results will be made accessible to the Clinical Pharmacologist involved with allocation and concealment of the participants at recruitment. In event necessitating calling off the trial, he will evoke the steering committee to stop the trial after extensive deliberation.

\section{Methods for additional analyses $\{20 \mathrm{~b}\}$}


Subgroup analysis will be performed using variables such as geopolitical region, gender, socio-economic status of parents and care provider, level of education and occupation.

\section{Methods in analysis to handle protocol non-adherence and any statistical methods to handle missing data $\{20 \mathrm{c}\}$}

The result of the study will be analysed per protocol. Missing data will be accounted for and the proportion with desirable outcome will be presented.

\section{Plans to give access to the full protocol, participant level-data and statistical code $\{31 \mathrm{c}\}$}

The protocol shall be published in a peered review journal and made publicly accessible to interested individuals or body. The participant level dataset and statistical code shall be made available after following due process adhering to good ethical standard.

\section{Oversight and monitoring}

\section{Composition of the coordinating centre and trial steering committee $\{5 \mathrm{~d}\}$}

A trial steering committee will consist of the principal investigator, two scientific enquirers, the public enquirer and a biostatistician. They will meet frequently to provide an oversight function for the trial conduct over the four centres in the country.

Each centre will have a hospital trial group headed by a consultant paediatrician who will be saddled with running daily events in the hospital, providing organizational support and reporting on a weekly basis to the steering committee. All investigators and monitors will be GCP compliant.

\section{Composition of the data monitoring committee, its role and reporting structure $\{21 a\}$}

The Data monitoring committee will consist of the Head of the Information Technology at the University College Hospital Ibadan. He will centrally manage the database. He will be supported by two assistants in event he is unable to perform his duties. They will be responsible for entering data from the UCH centre to the database. The Head will give access to focal persons (information technologist) at the other three collaborating centres in Nigeria. These individuals will be responsible for entering data into the central database in $\mathrm{UCH}$. Regular Zoom meeting will be held among the group members to address pressing issues. The data monitoring committee shall be independent of the core trial committee.

\section{Adverse event reporting and harms $\{22\}$}

All participants in this trial would be monitored for medication side effects and adverse drug reaction to the pharmarcovigilant group of the hospital. Any other unintended effects of trial interventions or trial conduct will be reported the hospital medical adversary committee. 
Frequency and plans for auditing trial conduct $\{23\}$

The local ethics board will monitor the progress of this trial and all medications and update will be relayed to the body as events unfold.

\section{Plans for communicating important protocol amendments to relevant parties $\{25\}$}

Any modification to the protocol or trial update will be communicated to the Ethical Approval bodies, Trial Registry and any other relevant parties.

\section{Dissemination plans $\{31$ a $\}$}

The outcome of this study will be communicated to participants, ethics board, healthcare professionals. It will be published in peer-reviewed scientific journals for public access. Data will be made available to the public maintaining ethical guidance.

\section{Discussion}

The outcome of this analysis will give insight into the efficacy of dual parenteral antimalarial in the treatment of cerebralal malaria among Nigerian children compared with standard of care. The safety profile of this intervention would also be highlighted. This may help inform Physicians on the optimal treatment for cerebral malaria to improve outcomes, prevent development of resistance, recrudescence, treatment failure and stimulate interest of pharmaceutical companies in the development of newer formulations of dual parenteral antimalarial combination therapies.

\section{Abbreviations}

RCT: Randomized Clinical Trial

PACTR: Pan Africa Clinical Trial Registry

GCMS: Glasglow Coma Score

GCP: Good Clinical Practice

MGCS: Modified Glasglow Coma Score

LMIC: Low And Middle-Income Countries

HIV: Human Immunodeficiency Syndrome

AIDS: Acquired Immunodeficienncy Syndrome 


\section{Declarations}

\section{Trial status}

The trial is active but not recruiting

\section{Acknowledgements}

We acknowledged the the children, parents and care providers who will voluntarily give consent to participate in this study. We are grateful to th Nurses and other Healthcare workers and senior colleagues in various hospitals involved in this study.

\section{Authors' contributions $\{31 \mathrm{~b}\}$}

A.M.A: The Principal Investigator; he conceived the study, led the proposal and protocol development. Critically revised the protocol for important intellectual content; gave final approval; agree to be accountable for all aspects of the work in ensuring that questions relating to the accuracy or integrity of any part of the work are appropriately investigated and resolved.

A.E.O Contributed to the design and development of the proposal and protocol; critically revised protocol; gave final approval; agrees to be accountable for all aspects of work ensuring integrity and accuracy

R.O.I. Contributed to the design and development of the proposal and protocol; critically revised protocol; gave final approval; agrees to be accountable for all aspects of work ensuring integrity and accuracy

A.0.0: Contributed to the design and development of the proposal and protocol; critically revised protocol; gave final approval; agrees to be accountable for all aspects of work ensuring integrity and accuracy.

A.O.A: Contributed to the design and development of the proposal and protocol; critically revised protocol; gave final approval; agrees to be accountable for all aspects of work ensuring integrity and accuracy.

D.A.G: Contributed to the design and development of the proposal and protocol; critically revised protocol; gave final approval; agrees to be accountable for all aspects of work ensuring integrity and accuracy.

I.J. 0: Contributed to the design and development of the proposal and protocol; critically revised protocol; gave final approval; agrees to be accountable for all aspects of work ensuring integrity and accuracy.

N.O.E Contributed to the design and development of the proposal and protocol; critically revised protocol; gave final approval; agrees to be accountable for all aspects of work ensuring integrity and accuracy.

All authors read and approved the final manuscript.

\section{Funding $\{4\}$}


This study is funded by the authors

\section{Availability of data and materials $\{29\}$}

Data and material from this study will be made available to the public unhindered. Individual Patient data will be de-identified and stored encrypted in a password-protected computer. The de-identified data will be publicly available at the trial website. De-identified data will also be stored in highly secured clouding computing.

\section{Ethics approval and consent to participate $\{24\}$}

Ethical approval was obtained from the State Research Ethics Review Committee (Ref:

NREC/12/04/2012) and the trial was also registered (PACTR202102893629864).

\section{Consent for publication $\{32\}$}

The Informed Consent Form will be redacted before sharing publicly. Patient identifiers will be removed from all documents for public access. The model consent form would be available on request through the corresponding author.

\section{Competing interests $\{28\}$}

The author(s) declared no potential conflicts of interest with respect to finance, conduct of the research, authorship, and/or publication of this protocol.

\section{Authors' information}

\section{Alao Michael Abe}

Department of paediatrics University College Hospital Ibadan and

Department of Paediatrics, Bowen University Teaching Hospital Ogbomoso

\section{Adebola Emmanuel Orimadegun}

Institute of Child Health, University of Ibadan

\section{Ibrahim Olayinka Rasheed}

Department of Paediatrics, Federal Medical Centre, Kastina, Kastina State, Nigeria

\section{Abayomi 0 Oyenuga}

University of Minnesota: Minneapolis, Minnesota, US

\section{Adanze Onyenonachi Asinobi}


Department of Paediatrics University College Hospital Ibadan

\section{Daniel Adedosu Gbadero}

Department of Paediatrics, Bowen University Teaching Hospital Ogbomoso

\section{Ifeoma Joy Okoye}

Department of Radiation Medicine, University of Nigeria Teaching Hospital, Enugu

\section{Emmanuel Okechukwu Nna}

The Molecular Pathology Institute

44 Rangers Avenue, Independence Layout, Enugu, Enugu State

\section{References}

1. White NJ, Pukrittayakamee S, Hien TT, Faiz MA, Mokuolu OA, Dondorp AM, "Malaria," (in eng), Lancet, vol. 383, no. 9918, pp. 723 - 35, Feb 22 2014, doi: 10.1016/s0140-6736(13)60024-0.

2. Crawley J, Chu C, Mtove G, Nosten F. "Malaria in children". The Lancet. 2010;375(9724):1468-81.

3. Luzolo AL, Ngoyi DM. "Cerebral malaria". Brain research bulletin. 2019;145:53-8.

4. Idro R, Marsh K, John CC, Newton CR. "Cerebral malaria: mechanisms of brain injury and strategies for improved neurocognitive outcome. Pediatr Res. 2010;68(4):267-74.

5. Postels DG, Birbeck GL. "Cerebral malaria". Handb Clin Neurol. 2013;114:91-102.

6. Shikani HJ, Freeman BD, Lisanti MP, Weiss LM, Tanowitz HB, Desruisseaux MS. "Cerebral malaria: we have come a long way". Am J Pathol. 2012;181(5):1484-92.

7. Sachs J, Malaney P, "The economic and social burden of malaria. NaY ture," ed: Nature Publishing Group, 2002.

8. Organization WH, World malaria report 2015. World Health Organization, 2016.

9. Organization WH, Guidelines for the treatment of malaria. World Health Organization, 2015.

10. Esu EB, Effa EE, Opie ON, Meremikwu MM. "Artemether for severe malaria" Cochrane Database of Systematic Reviews, no. 6, 2019.

11. Organization WH, Global technical strategy for malaria 2016-2030. World Health Organization, 2015.

12. A Kumar et al. Early combination antibiotic therapy yields improved survival compared with monotherapy in septic shock: a propensity-matched analysis. Critical care medicine. 2010;38(9):1773-85.

13. Kumar A, Safdar N, Kethireddy S, Chateau D. "A survival benefit of combination antibiotic therapy for serious infections associated with sepsis and septic shock is contingent only on the risk of death: a 
meta-analytic/meta-regression study". Critical care medicine. 2010;38(8):1651-64.

14. RB Mokhtari et al., "Combination therapy in combating cancer," Oncotarget, vol. 8, no. 23, p. 38022, 2017.

15. I Coquet et al. "Survival trends in critically ill HIV-infected patients in the highly active antiretroviral therapy era". Crit Care. 2010;14(3):1-9.

16. "Nigeria Health Facility Registry accessed on 04/03/2021 from; https://hfr.health.gov.ng/facilities/hospitals-list?page $=8$ ".

17. "Federal Ministry of Health;List of all Federal Teaching Hospitals. Acced on 04/03/2021; https://www.health.gov.ng/index.php?option=com_content\&view=article\&id=136\&ltemid=502.".

18. Chan AW, Tetzlaff JM, Altman DG, Laupacis A, Gøtzsche PC. Krleža- Jerić K, et al SPIRIT 2013 statement: dening standard protocol items for.

\section{Figures}




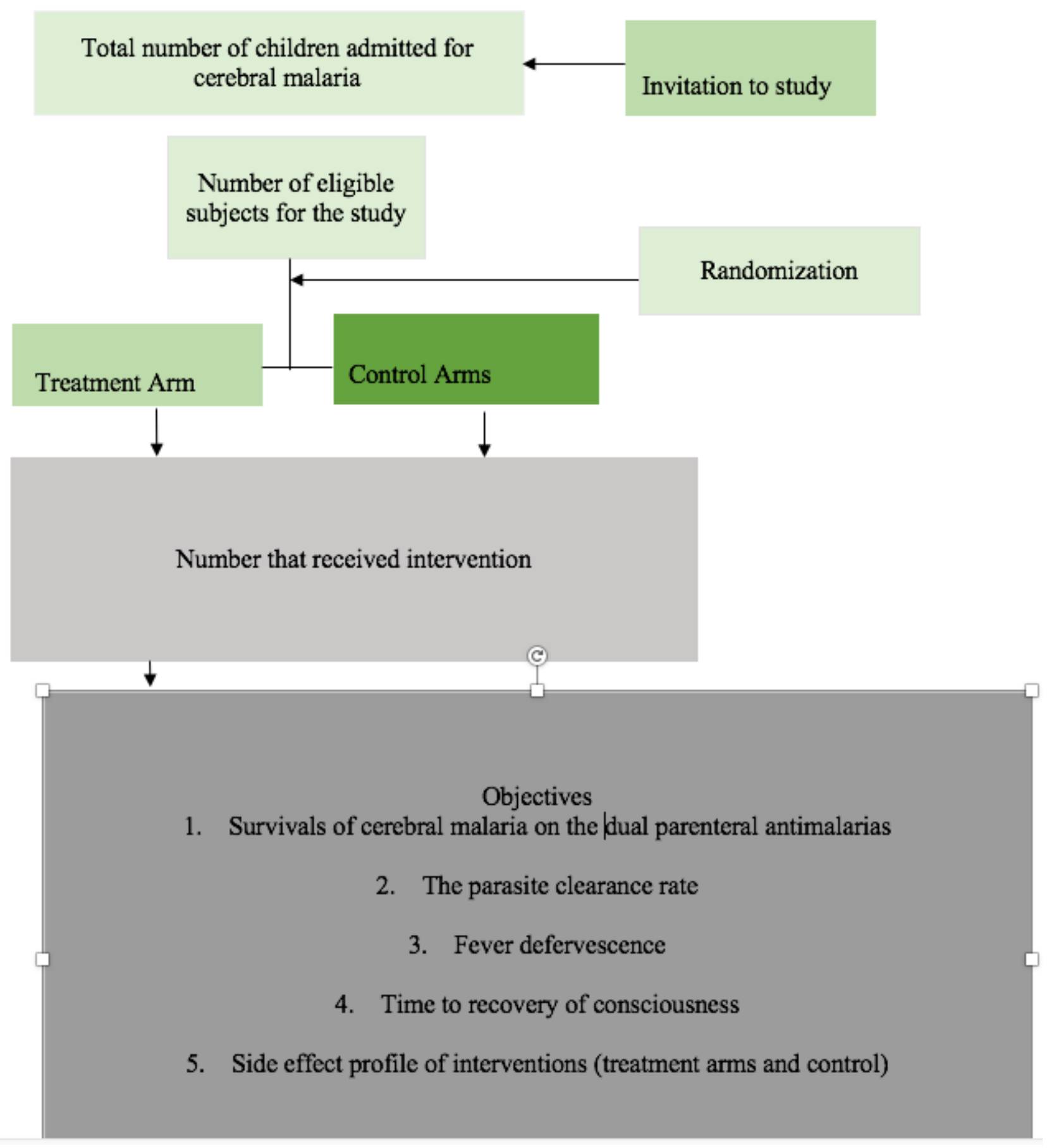

\section{Figure 1}

The participants' flow chart from invitation through enrolment, allocation to trial. 


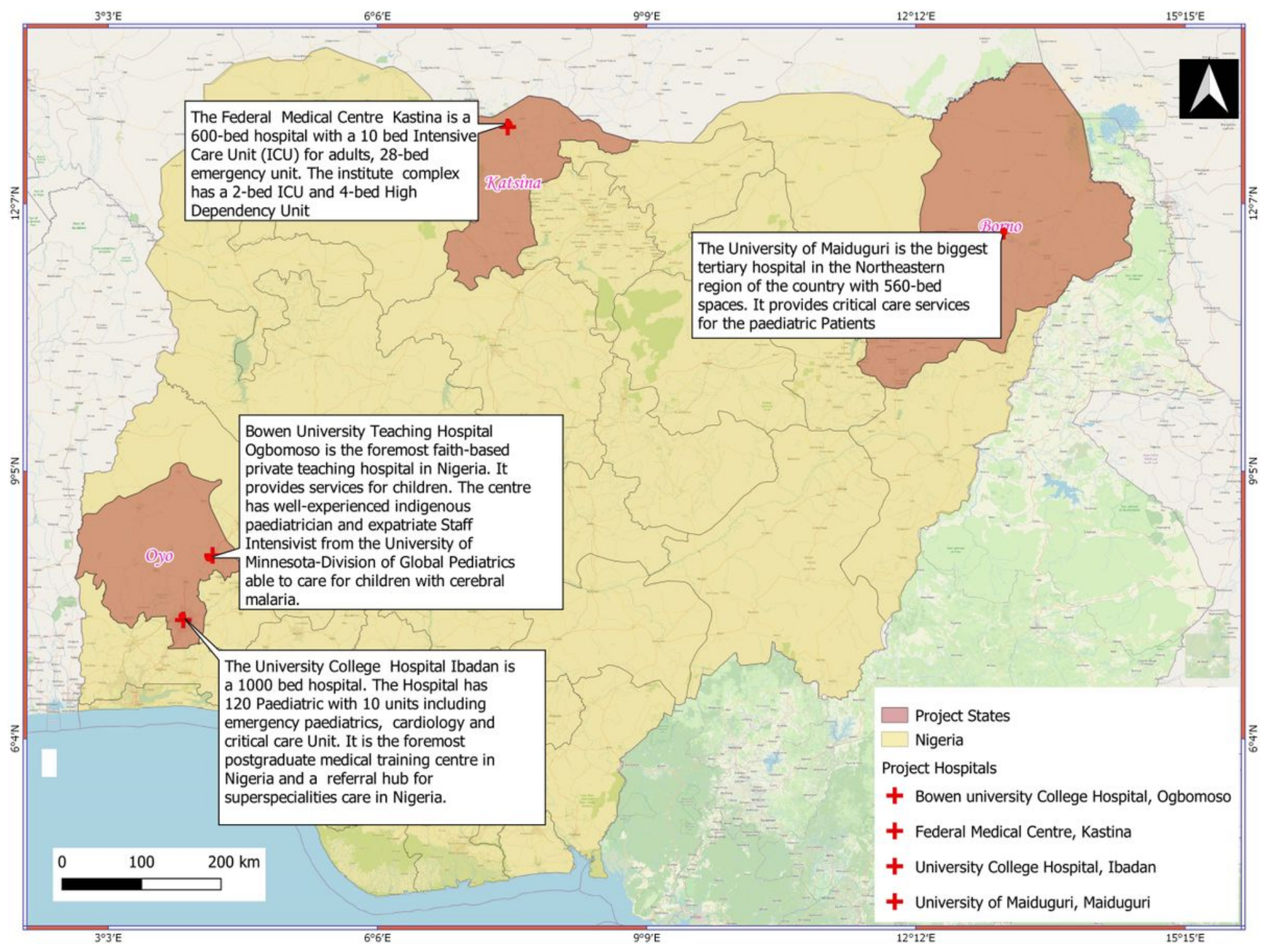

Figure 2

This study is a multicenter study involving 4 tertiary hospitals (one private and three public) located in three of the six geopolitical regions in Nigeria.[16, 17] The selected tertiary hospital includes University College Hospital Ibadan. Bowen University Teaching Hospital Ogbomoso, The Federal Medical Centre Kastina, Kastina state and The University of Maiduguri Teaching Hospital, Maiduguri. Note: The designations employed and the presentation of the material on this map do not imply the expression of any opinion whatsoever on the part of Research Square concerning the legal status of any country, territory, city or area or of its authorities, or concerning the delimitation of its frontiers or boundaries. This map has been provided by the authors. 